\title{
Tricuspid Valve Replacement: Mechanical or Biological Prostheses? A Systematic Review and Meta-Analysis
}

\author{
Zeyi Cheng, MD, ${ }^{1}$ Tingting Fang, $\mathrm{PhD},{ }^{2}$ Dandan Wang, PhD,${ }^{3}$ Yingqiang Guo, $\mathrm{MD}^{1}$ \\ ${ }^{1}$ Department of Cardiovascular Surgery, West China Hospital, Sichuan University, No. 37, Guoxue Alley, Chengdu, Sichuan 610041, \\ China; ${ }^{2}$ Department of Cardiology, West China Hospital, Sichuan University, Chengdu, Sichuan 610041, China; ${ }^{3}$ The First School of \\ Clinical Medicine, Lanzhou University, Lanzhou 730000, China
}

\section{ABSTRACT}

Background: Tricuspid valve replacement (TVR) is seldom performed in cardiac valve surgery, and there currently are no clinical guidelines as to which type of prostheses is better in tricuspid valve position. This meta-analysis was performed to compare the results of mechanical and biological prostheses for TVR.

Methods: We searched the Pubmed, Cochrane, and Embase clinical trial databases to collect all related studies published from January 1, 2000 to July 31, 2020. A randomeffects model was used to evaluate the odds ratios (OR) and its $95 \%$ confidence intervals (CI) of time-to-event related effects of the surgical procedures; every study's quality was evaluated by the Newcastle-Ottawa Scale (NOS).

Results: A total of 13 retrospective studies, including 1453 patients were analyzed. There were no statistically differences between mechanical and biological prostheses with respect to prosthetic valve failure $[\mathrm{OR}=0.84,95 \% \mathrm{CI}(0.54,1.28)$, $P=.41]$, bleeding $[\mathrm{OR}=0.84,95 \% \mathrm{CI}(0.54,1.28), P=.41]$, reoperation $[\mathrm{OR}=1.02,95 \% \mathrm{CI}(0.58,1.78), P=.95]$, early mortality $[\mathrm{OR}=1.35,95 \% \mathrm{CI}(0.82,2.25), P=.24]$ and longtime survival $[\mathrm{OR}=1.09,95 \% \mathrm{CI}(0.70,1.69), P=.70]$, but a significant difference can be seen in mechanical prostheses with a higher risk of thrombosis $[\mathrm{OR}=0.17,95 \% \mathrm{CI}(0.05$, $\left.0.60), P=.006, \mathrm{I}^{2}=0 \%\right]$.

Conclusions: In tricuspid valve position, mechanical valve prostheses have a higher risk of thrombosis than biological prostheses, but no statistical differences between mechanical and biological prostheses with respect to prosthetic valve failure, bleeding, reoperation, early mortality, and long-term survival. The valve disease and patient's age and risk factors are the most important considerations in the decision-making process. The more specific conclusion needs to be further proved by large-sample, multi-center, randomized, double-blind and control trials.

Received December 9, 2020; received in revised form December 27, 2020; accepted fanuary 3, 2021.

Correspondence: Yingqiang Guo, Department of Cardiovascular Surgery, West China Hospital, Sichuan University, No. 37, Guoxue Alley, Wubou District, Chengdu, Sichuan 610041, China; +86-18980601876 (e-mail: drguoyq@hotmail.com).

\section{INTRODUCTION}

TVR procedure accounts for less than $10 \%$ of all interventions on the tricuspid valve [Rizzoli 1998; Ratnatunga 1998]. Most TVR cases can get satisfactory effects via implant, prosthetic ring, or tricuspid annuloplasty [Calafiore 2011; McCarthy 2004]. Replacement of the tricuspid valve is a necessary measure in cases where annuloplasty is not possible (infective endocarditis, Ebstein's anomaly, reoperation after unsuccessful plastic surgery, etc.). TVR mostly is given full consideration by the surgeon as the final treatment method [Rizzoli 1998]. Mechanical prostheses generally are considered better durability, but relate to thrombosis, bleeding events caused by anticoagulation, and decreased turbulence [Rizzoli 1998; Péterffy 2001; Kaplan 2002]. Biological prostheses do not require long-term anticoagulation therapy and reduce hemorrhagic events, but they are related to limited durability and structural valve deterioration [Chang 2006]. Currently, no specific guidelines recommend the type of prosthetic valve that will be the best choice in the tricuspid position [Vahanian 2012]; it mostly depends on the attending surgeon's personal preference [Songur 2014].

The purpose of this meta-analysis is aimed at analyzing the early and long-term outcomes of mechanical and biological prostheses replacement options and help decide which type is the best option for patients who need TVR.

\section{PATIENTS AND METHODS}

This meta-analysis was conducted in accordance with the Preferred Reporting Items for Meta-Analyses (PRISMA) statement to the Quality of Reporting of Meta-Analyses Statement [Moher 2009]. The work has been reported in line with PRISMA (Preferred Reporting Items for Systematic Reviews and Meta-Analyses) and AMSTAR (Assessing the methodological quality of systematic reviews) Guidelines. The research protocol was registered in researchregistry (ID: reviewregistry993).

Search strategy and data extraction: Literature search was performed through the Pubmed, Cochrane, and Embase clinical trial databases to collect related studies published from January 1, 2000 to July 31, 2020. The following search terms were used: 1) "tricuspid valve"; 2) "replantation" OR "reimplantation" OR "replacement" OR "substitution"; 3) "surgery" 
Table 1. The basic characteristics

\begin{tabular}{|c|c|c|c|c|c|c|c|c|}
\hline Liang WT & 2019 & China & 43 & 33 & 76 & $32.89 \%$ & 45.7 & $43.3 \pm 21.9$ months \\
\hline $\begin{array}{l}\text { Redondo } \\
\text { Palacios A }\end{array}$ & 2017 & Spain & 81 & 29 & 110 & $39.53 \%$ & 63.4 & mean 7 years \\
\hline Connolly HM & 2015 & America & 159 & 36 & 195 & $50.3 \%$ & 61 & 10 years \\
\hline Songur CM & 2014 & Turkey & 68 & 64 & 132 & $34.7 \%$ & 61 & 12 years \\
\hline Young & 2013 & Korea & 37 & 55 & 31 & $55.2 \%$ & 32 & $1-235$ months \\
\hline $\begin{array}{l}\text { Rodríguez- } \\
\text { Capitán J }\end{array}$ & 2013 & Spain & 11 & 24 & 35 & $25 \%$ & 55.9 & 41 months \\
\hline Garatti A & 2012 & Italy & 44 & 46 & 90 & $38.5 \%$ & 53.8 & $1-28$ years \\
\hline Young $\mathrm{H}$ & 2012 & Korea & 49 & 70 & 119 & $30.8 \%$ & 53 & $68 \pm 38$ months \\
\hline
\end{tabular}

OR “operation" OR "surgical procedures, operative" OR "operative surgical procedures" OR "general surgery." Search strategies were: Search "Tricuspid Valve"[Mesh] OR tricuspid valve [Title/Abstract] OR tricuspid valves [Title/Abstract] AND "Surgical Procedures, Operative"[Mesh] OR surgery [Title/Abstract] OR operation [Title/Abstract] OR operative surgical procedures [Title/Abstract] OR general surgery [Title/Abstract] AND "Replantation"[Mesh] OR reimplantation [Title/Abstract] OR replacement [Title/Abstract] OR substitution [Title/Abstract]. The literature obtained was carefully reviewed by the inclusion and exclusion criteria. All papers were limited to English language.

Selection criteria and data extraction: Papers must include comparative data for both mechanical and biological valves replacement surgery, RCT articles. The exclusion criteria were: 1) No direct comparison of biological and mechanical valves in the studies; 2) Comparison of tricuspid valve repair and tricuspid valve replacement; 3) No related survival data in the article; 4) The tricuspid valve replacement was not the only intervention on the heart; 5) Letters, case reports, comments, and meeting abstracts. Data extraction was independently conducted by two authors, using a standardized data collection form. Differences were decided by the senior author. Early mortality was defined as death within the first 30 postoperative days in all studies.

Assessment of risk of bias: We used the Newcastle-Ottawa Scale (NOS), which was recommended by the Cochrane Manual to evaluate the risk of bias of all included studies. Scores $\leq 5$ were categorized as a high risk of bias, while $>5$ was considered a low risk of bias. The results were carefully compared, and disagreements were resolved by discussion.

Statistical analysis: Due to the good homogeneity of the included studies, a fixed-effect model was used for each outcome (prosthetic valve failure, bleeding, reoperation, early mortality, and long-term survival). The outcome events were assessed by OR and $95 \% \mathrm{CI}, \mathrm{I}^{2}$ was used to evaluate the heterogeneity of effect, $\mathrm{I}^{2}$ values were less than $25 \%$, low heterogeneity, $\mathrm{I}^{2}$ between $25-50 \%$, moderate heterogeneity; and $\mathrm{I}^{2}$ greater than $50 \%$, substantial heterogeneity [Higgins 2003]. Funnel plots were used to assess publication bias when necessary [Easterbrook 1991]. Statistical analyses were conducted by Review Manager (Version 5.3 Cochrane Collaboration, Software Update, Oxford, United Kingdom).

\section{RESULTS}

Based on our search terms and search strategies, the search results were shown in Figure 1 in line with the PRISMA flow chart. (Figure 1) A total of 13 studies [Songur 2014; Liang 2019; Wiedemann 2018; Redondo Palacios 2017; Hwang 2016; Mao 2016; Connolly 2015; Hwang 2014; Rodríguez-Capitán 2013; Cho 2013; Altaani 2013; Garatti 2012; Hwang 2012] with 1453 patients were analyzed, of which 793 patients received biological prostheses, while 660 patients received mechanical prostheses. Individual characteristics of the studies can be seen in Table 1. All final selected literature were case control retrospective studies. We used the NOS recommended by the Cochrane Manual to evaluate all studies. The quality NOS scores of each study are shown in Table 2.

Reoperation: Ten studies [Songur 2014; Liang 2019; Wiedemann 2018; Redondo Palacios 2017; Connolly 2015; Hwang 2014; Rodríguez-Capitán 2013; Cho 2013; Altaani 2013; Hwang 2012] reported the reoperation rate and were included in the meta-analysis. The pooled OR estimate was 1.02 ( 0.58 to $1.78, P=.95, \mathrm{I}^{2}=0 \%$ ) (Figure $\left.2 \mathrm{~A}\right)$. The results showed the studies have good homogeneity, but no statistical 


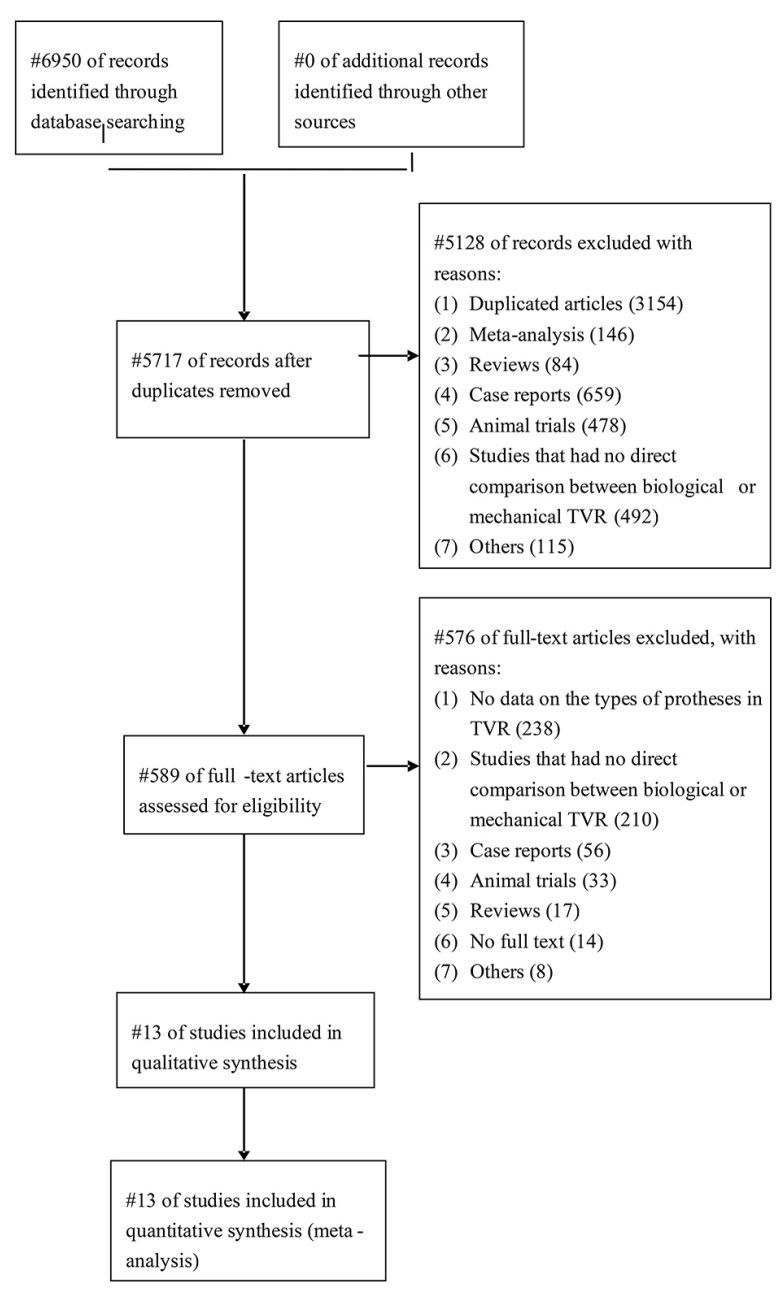

Figure 1. Literature screening process.

significance in this meta-analysis. Regardless of mechanical or biological prostheses after TVR, there is no difference in the reoperation rate. In these studies, the reoperation rate of biological valves was $1.94 \%-22 \%$ while mechanical prostheses were $0.83 \%-19.57 \%$.

Early mortality: Seven studies [Liang 2019; Redondo Palacios 2017; Hwang 2014; Cho 2013; Altaani 2013; Garatti 2012; Hwang 2012] reported data on early deaths after TVR. The pooled OR estimate was $1.35\left(0.82\right.$ to $2.25, P=.24, \mathrm{I}^{2}=$ $35 \%$ ) (Figure 2B). The results suggested no significant difference. Early mortality after TVR was $0-23.46 \%$ for biological valves and $3.03 \%-40 \%$ for mechanical valves respectively.

Long-term survival: Seven studies [Liang 2019; Wiedemann 2018; Redondo Palacios 2017; Rodríguez-Capitán 2013; Cho 2013; Altaani 2013; Garatti 2012] provided longterm survival data, the pooled OR estimate was 1.09 (0.70 to $1.69, P=0.70, \mathrm{I}^{2}=0 \%$ ) (Figure $2 \mathrm{C}$ ). The results showed the studies have good homogeneity, but no statistical significance in this meta-analysis. These seven published articles reported long-term survival data. The 5 -year, 10 -year, and 15 -year survival rates after biological prosthetic valve replacement were
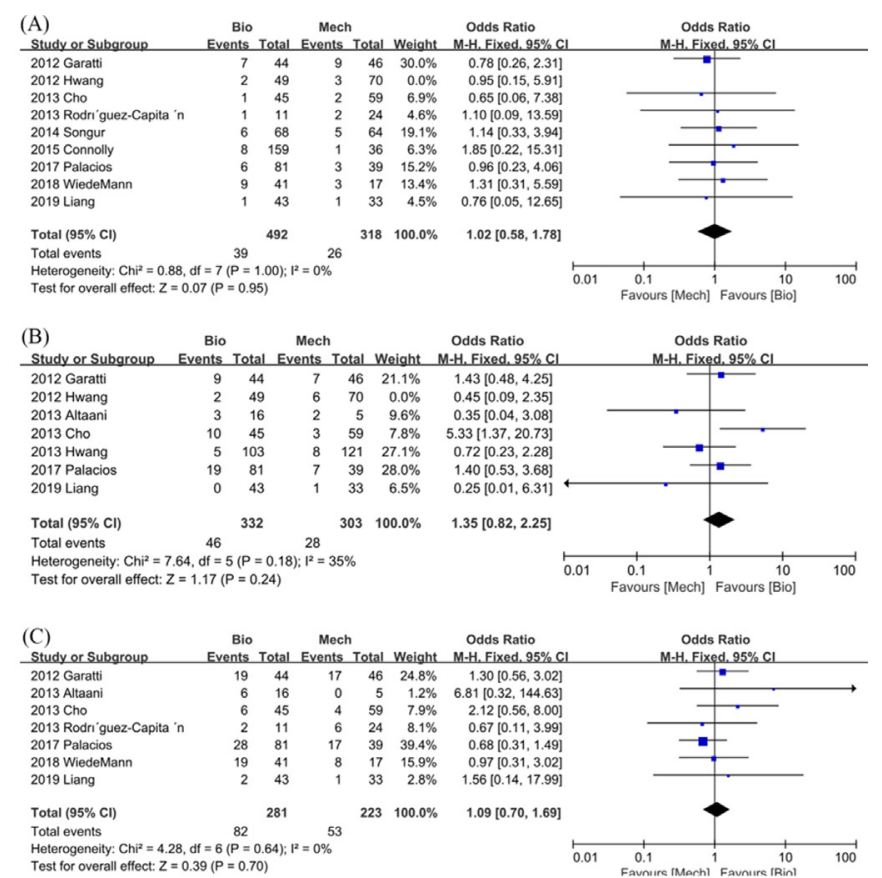

Figure 2. Survival hazard ratio of each study with related $95 \%$ confidence limits. Small significant heterogeneity was found. A) Reoperation. The pooled OR estimate was $1.02\left(0.58\right.$ to $\left.\left.1.78, P=.95, \mathrm{I}^{2}=0 \%\right) . \mathrm{B}\right)$ Early mortality. The pooled OR estimate was 1.35 (0.82 to 2.25, $P=$ $\left..24, I^{2}=35 \%\right)$. C) Long-term survival. The pooled OR estimate was 1.09 $\left(0.70\right.$ to $\left.1.69, P=.70, I^{2}=0 \%\right)$.

$26.76 \%-81.63 \%, 2.08 \%-72.45 \%$, and $0-58.89 \%$, respectively. For mechanical prostheses, the 5 -year, 10-year, and 15 -year survival rates were $24.32 \%-94.12 \%, 5.56 \%-81.36 \%$, $0-67.80 \%$, respectively. The difference in results mainly is due to the inconsistent follow-up time and four studies did not report the Kaplan-Meier curves.

Thromboembolism: Six studies [Songur 2014; Liang 2019; Connolly 2015; Hwang 2014; Cho 2013; Hwang 2012] provided data on thrombosis. The pooled OR estimate was $0.17\left(0.05\right.$ to $\left.0.60, P=0.006, \mathrm{I}^{2}=0 \%\right)$ (Figure $\left.3 \mathrm{~A}\right)$. The results were statistically significant, and the homogeneity of the literature was good. Mechanical prostheses are more prone to thrombosis than bioprostheses. The incidence of postoperative thrombosis for the mechanical and biological valve was $1.69 \%-7.81 \%$ and $0-2.20 \%$, respectively.

Anticoagulant-related bleeding: Seven studies [Songur 2014; Liang 2019; Redondo Palacios 2017; Hwang 2014; Rodríguez-Capitán 2013; Cho 2013; Hwang 2012] reported anticoagulant-related bleeding data. The pooled OR estimate was 0.84 ( 0.54 to $1.28, P=0.41, \mathrm{I}^{2}=26 \%$ ) (Figure $3 \mathrm{~B}$ ). There was no statistical significance. The incidence of anticoagulation-related bleeding events in patients with biological and mechanical valves were $0-10.29 \%$ and $3.03 \%-18.57 \%$, respectively.

Prosthetic valve failure: Three articles [Liang 2019; Redondo Palacios 2017, Cho 2013] reported data on biological prosthetic failure, and the pooled OR estimate was 0.84 ( 0.54 to $1.28, P=0.41, \mathrm{I}^{2}=26 \%$ ) (Figure $3 \mathrm{C}$ ). There 

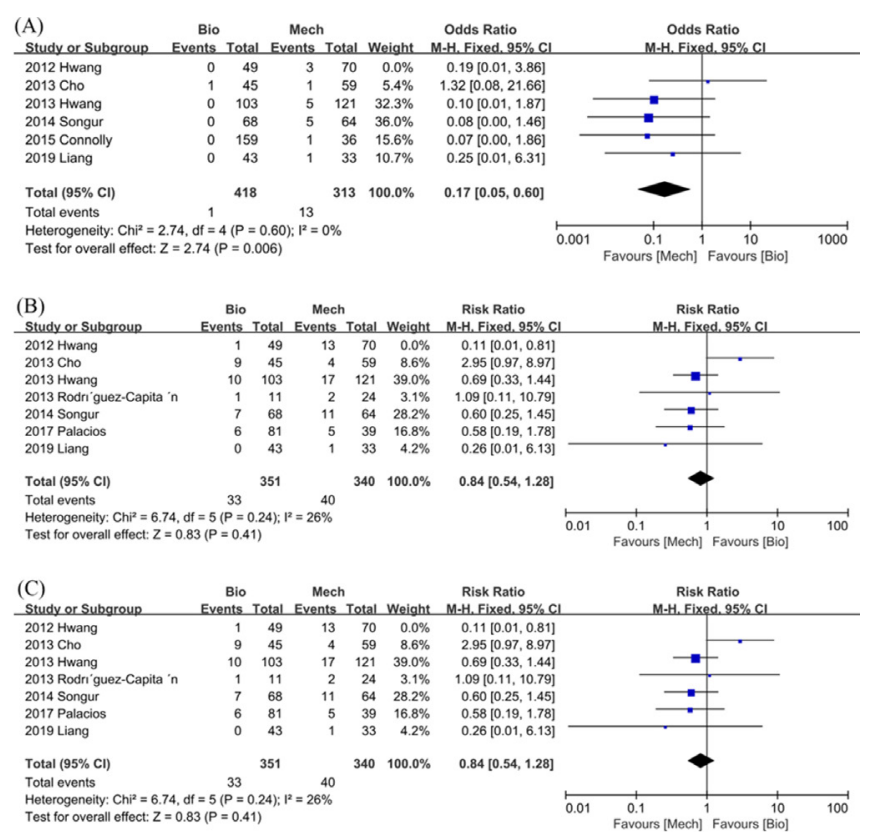

Figure 3. Survival hazard ratio of each study with related $95 \%$ confidence limits. Small significant heterogeneity was found. A) Thromboembolism. The pooled OR estimate was 0.17 (0.05 to 0.60 , $\left.P=.006, I^{2}=0 \%\right)$. B) Anticoagulant-related bleeding. The pooled OR estimate was $0.84\left(0.54\right.$ to $\left.1.28, P=.41, I^{2}=26 \%\right)$. C) Prosthetic failure. The pooled OR estimate was $0.84\left(0.54\right.$ to $\left.1.28, P=0.41, I^{2}=26 \%\right)$.

was no statistical significance, and the homogeneity of the literature was good. The mechanical valve and biological prosthetic valve failure rates were $5.08 \%-12.12 \%$ and $7.4 \%-18.60 \%$, respectively.

\section{DISCUSSION}

There still is ongoing controversy as to the type of prosthetic to choose in the tricuspid position [McCarthy 2004; González-Santos 2013]. Mechanical prosthetic valves possess good durability and lower gradients, but there is a risk of prosthetic thrombosis and anticoagulation-related bleeding events. Patients need to receive lifelong anticoagulation therapy, while biological prosthetic valves do not require lifelong anticoagulation, but the prosthetic's lifespan is not as long as a mechanical valve. For calcification of the prosthesis, structural failure, especially in young patients, they always need to undergo redo TVR, which is related to high morbidity and mortality.

Rizzoli et al [Rizzoli 2004] conducted a meta-analysis, reviewing studies published from 1995 to 2004; 1160 patients were analyzed. Liu et al [Liu 2016] also analyzed the literature and selected 22 studies, including 2630 patients in their meta-analysis. Their findings almost were similar. Considering this point and the improvement of surgical technology and prosthetic valve quality as well as medical standards in recent years, we searched the literature for the latest 10 years to compare progress in TVR in recent years with previously published studies.

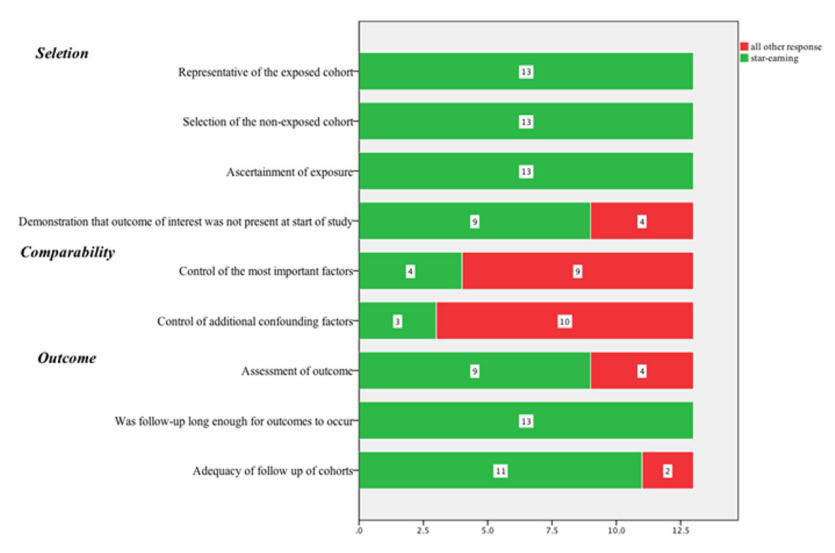

Table 2. Risk of bias of the included studies using the Newcastle-Ottawa Scale (NOS)

Reoperation is an important consideration for attending surgeons deciding whether to use a mechanical or biological prosthetic valve at the tricuspid position. The higher rate of mechanical valve thrombosis in the tricuspid valve position can be interpreted by the lower pressure of the right heart system and the right ventricular morphology, as well as the low prostacyclin concentration of venous blood. Songur et al [Songur 2014] reported the whole reoperation rate was $8.3 \%$, and reoperation was performed on average 44.2 months after mechanical valve replacement. The main reason was not taking warfarin strictly, which lead to valve thrombosis, while reoperation was performed on average 92.3 months after biological prostheses replacement, primarily for valve degeneration. Similarly, Hwang et al [Hwang 2012] also reported in the group that received a mechanical valve replacement, only one patient underwent reoperation after 58 months due to thrombosis. However, according to González-Santos et al [González-Santos 2013], after 7-10 years, the reoperation rate of the tricuspid valve was higher. Although there is no significant statistical difference, mechanical prosthetic valves have a higher incidence of thrombosis and an earlier risk of reoperation. This phenomenon could be explained via the different follow-up times of the two complications. Therefore, patients receiving mechanical valves should focus on the management of anticoagulation after surgery, and for patients receiving biological valves, the factors of valve failure should be reduced or even avoided, such as control of blood lipids, blood pressure and blood sugar, make reasonable anticoagulation management and so on.

Redondo Palacios et al [Redondo Palacios 2017] observed that postoperative (excluding one endocarditis-related patient) valve failure occurred more often in patients with bovine pericardial prostheses than with porcine prostheses. The main reason for this phenomenon: Bovine bioprostheses have a closing volume, which is a small central regurgitation jet for the stiff leaflets. Similarly, Mayo Clinic prefers porcine prostheses in TVR, Dearani [Dearani 2014] explained, "In right heart, the relatively thicker and stiffer pericardial leaflets do not open and close properly with the low opening 
and low closing pressures often combined with depressed right ventricle function." However, there is currently no clinical research on the selection of bovine pericardium or porcine pericardium prostheses specifically for the tricuspid valve position.

With the improvement of surgical techniques, redo tricuspid valve operation with a simplified, minimally invasive, beating-heart technique was both feasible and safe [Dearani 2014].

Regardless of choosing a mechanical valve or bioprosthetic valve, the early postoperative mortality rate is equivalent, and there is no statistical difference. Meanwhile, there is no difference in early mortality between those undergoing TVR on cardiopulmonary bypass (CPB) with the heart beating and those operated on under cardioplegic arrest [Altaani 2013]. A high operative mortality rate between $12 \%-26 \%$ and this ratio is relatively constant, according to recently published studies [Dearani 2014; Filsoufi 2005; Nakano 2001]. Previous studies have shown large perioperative furosemide dose is associated with higher operative mortality [Hwang 2016]; postoperative low-output syndrome and liver congestion with hepatic serum enzymes values (transaminases and/or total bilirubin) two times higher than normal value were independent predictors of in-hospital mortality [Altaani 2013]. Both of those factors indicated when the right heart function affects the systemic blood circulation and causes the related organs (hepatic, kidney, etc.) dysfunction, the risk of early death is very high. It is clear right heart failure is the main cause of death in the current published studies, $i$, and this is also unanimously recognized by cardiac experts.

Long-term mortality after TVR surgery has nothing to do with valve types, Palacios [Wiedemann 2018] reported the long-term mortality in mechanical and biological prostheses were $43.60 \%$ and $34.60 \%$, respectively, without a significant difference $(P=.31)$. The type of implanted valve was not significantly associated with late mortality through logistic regression analysis $(P=.31)$. There was no statistical significance in survival at 5,10 , and 15 years. Survival was $73 \%-83 \%, 67 \%-81 \%$, and $63 \%-76 \%$, respectively, in the mechanical group and $70 \%-78 \%, 60 \%-75 \%$, and $57 \%-$ $68 \%$, respectively, in the bioprostheses group [Hwang 2016; Altaani 2013].

In this meta-analysis, mechanical prostheses were more prone to thrombosis than bioprostheses in the tricuspid valve position. This is consistent with previous studies. Mechanical valve thrombosis occurs more frequently in the first years postoperatively, and its incidence decreases 5 to 7 years after the operation. Rizzoli et al [Rizzoli 2004] reported that bioprosthetic valve degeneration increased at a steeper rate 7 years after operation. This also was confirmed by Garatti, as valve thrombosis occurred at a mean follow-up of 6 years and structural valve degeneration happened at an average of 9 years after TVR [Garatti 2012].

Transcatheter tricuspid valve intervention (TTVI) for tricuspid valve dysfunction has been applied in recent years as an alternative therapeutic option to serve a large highrisk population of patients undergoing severe symptomatic tricuspid regurgitation (TR). Although clinical data are limited regarding the efficacy of TTVI to date, feasibility has been proven by different techniques, including annuloplasty devices, leaflet and coaptation devices, and valve replacement, both in the heterotopic [Rosser 2016; Nickenig 2017] to reduce the backflow in the venous-system and the orthotropic positions. TTVI would be a high impact strategy for select high-risk patients with TR with diseased leaflets. This ultimately may help to avoid a high-risk operation in patients with highly co-morbidity conditions, including valve in valve surgery, which contributes to those high-risk patients who need tricuspid valve surgery for the second or even third time.

\section{CONCLUSION}

In TVR surgery, the choice between a biological valve and mechanical valve still is inconclusive. This meta-analysis strongly indicates the risk of thrombosis in mechanical valves is higher. The remaining complications are not significantly different between the two types of valves in our meta-analysis and previously published studies. Although there are no gold standard guidelines for the type of prosthetic valve that should be selected in the tricuspid position, valve disease and age are the most important factors that should be taken into consideration during the decision-making process. RCTs and multicenter research still need to be performed to decide which prosthetic valve type would be better for TVR.

\section{LIMITATIONS}

There are several inevitable limitations in this metaanalysis. First, we selected the last 20 years of studies, and the selection bias was inevitable. Second, all selected studies are retrospective. Third, since there is no unified standard, the choice of prosthetic valves for tricuspid valve replacement in each heart center depends on the surgeon's preference [Vahanian 2012]. Studies involved in the analysis have a relatively short follow up. The conclusion of this meta-analysis should be interpreted carefully, and more importantly, RCT must be performed to decide which prosthetic valve type is better for TVR.

\section{REFERENCES}

Altaani HA, Jaber S. 2013. Tricuspid Valve Replacement, Mechnical vs. Biological Valve, Which Is Better? Int Cardiovasc Res J. 7(2):71-4.

Calafiore AM, Iaco AL, Romeo A, et al. 2011. Echocardiographicbased treatment of functional tricuspid regurgitation. J Thorac Cardiovasc Surg. 142: 308-13.

Chang BC, Lim SH, Yi G, et al. 2006. Long-term clinical results of tricuspid valve replacement. Ann Thorac Surg. 81-(4):1317-1323, discussion 23-4.

Cho WC, Park CB, Kim JB, et al. 2013. Mechanical valve replacement versus bioprosthetic valve replacement in the tricuspid valve position. J Card Surg. 28(3):212-7. 
Connolly HM, Schaff HV, Abel MD, et al. 2015. Early and Late Outcomes of Surgical Treatment in Carcinoid Heart Disease. J Am Coll Cardiol. 66(20):2189-96.

Dearani JA. 2014. Editorial comment: lessons learned with tricuspid valve replacement. eur J Cardiothorac surg. 45:90-1.

Easterbrook PJ, Berlin JA, Gopalan R, et al. 1991. Publication bias in clinical research. Lancet (London, England). 337(8746):867-72.

Filsoufi F, Anyanwu AC, Salzberg SP, et al. 2005. Long-term outcomes of tricuspid valve replacement in the current era. Ann Thorac Surg. $80: 845-50$.

Garatti A, Nano G, Bruschi G, et al. 2012. Twenty-five years outcomes of tricuspid valve replacement comparing mechanical and biologic prostheses. Ann Thorac Surg. 93(4):1146-53.

González-Santos JM, Arnáiz-García ME. 2013. Correcting tricuspid regurgitation: an unresolved issue. Rev Esp Cardiol (Engl Ed). 66: 609-12.

Higgins JPT, Thompson SG, Deeks JJ, et al. 2003. Measuring inconsistency in meta-analyses. BMJ. 327(7414):557-60.

Hwang HY, Kim KH, Kim KB, et al. 2012. Mechanical tricuspid valve replacement is not superior in patients younger than 65 years who need long-term anticoagulation. Ann Thorac Surg. 93(4):1154-60.

Hwang HY, Kim KH, Kim KB, et al. 2014. Propensity score matching analysis of mechanical versus bioprosthetic tricuspid valve replacements. Ann Thorac Surg. 97(4):1294-9.

Hwang HY, Kim KH, Kim KB, et al. 2016. Reoperations after tricuspid valve repair: re-repair versus replacement. J Thorac Dis. 8(1):133-9.

Kaplan M, Kut MS, Demirtas MM, et al. 2002. Prosthetic replacement of tricuspid valve: Bioprosthetic or mechanical. Ann Thorac Surg. 73: 467-73.

Liang W, Yue H, Li T, et al. 2019. The better substitute for tricuspid valve replacement in patients with severe isolated tricuspid regurgitation. Anatol J Cardiol. 22(4):172-6.

Liu P, Qiao WH, Sun FQ, et al. 2016. Should a Mechanical or Biological Prosthesis Be Used for a Tricuspid Valve Replacement? A Meta-Analysis. J Card Surg. 31(5):294-302.

Mao B, Sun L, Zhang J, et al. 2016. Perioperative factors associated with short- and long-term outcomes after tricuspid valve replacement. Interact Cardiovasc Thorac Surg. 23(6):845-50.

McCarthy PM, Bhudia SK, Rajeswaran J, et al. 2004. Tricuspid valve repair: durability and risk factors for failure. J Thorac Cardiovasc Surg. 127: 674-85.
Moher D, Liberati A, Tetzlaff J, et al. 2009. Preferred reporting items for systematic reviews and meta-analyses: the PRISMA statement, BMJ 339. b2535.

Nakano K, Ishibashi-Ueda H, Kobayashi J, et al. 2001. Tricuspid valve replacement with bioprostheses: long-term results and causes of valve dysfunction. Ann Thorac Surg. 71:105-9.

Nickenig G, Kowalski M, Hausleiter J, et al. 2017. Transcatheter treatment of severe tricuspid regurgitation with the edge-to-edge MitraClip technique. Circulation. 135:1802-14.

Péterffy A, Szentkirályi I. 2001. Mechanical valves in tricuspid position: cause of thrombosis and prevention. Eur J Cardiothorac Surg. 19:735-6.

Ratnatunga CP, Edwards MB, Dore CJ, et al. 1998. Tricuspid valve replacement: UK Heart Valve Registry mid-term results comparing mechanical and biological prostheses. Ann Thorac Surg. 66:1940-7.

Redondo Palacios A, López Menéndez J, Miguelena Hycka J, et al. 2017. Which type of valve should we use in tricuspid position? Long-term comparison between mechanical and biological valves. J Cardiovasc Surg (Torino). 58(5):739-46.

Rizzoli G, De Perini L, Bottio T, et al. 1998. Prosthetic replacement of the tricuspid valve: biological or mechanical? Ann Thorac Surg. 66(Suppl):62-7.

Rizzoli G, Vendramin I, Nesseris G, et al. 2004. Biological or mechanical prostheses in tricuspid position? A meta-analysis of intra-institutional results. Ann Thorac Surg 77(5):1607-14.

Rodríguez-Capitán J, Gómez-Doblas JJ, Fernández-López L, et al. 2013. Short- and long-term outcomes of surgery for severe tricuspid regurgitation. Rev Esp Cardiol (Engl Ed). 66(8):629-35.

Rosser BA, Taramasso M, Maisano F. 2016. Transcatheter interventions for tricuspid regurgitation: TriCinch (4Tech). EuroIntervention. 12:Y110-2.

Songur CM, Simsek E, Ozen A, et al. 2014. Long-term results comparing mechanical and biological prostheses in the tricuspid valve position: which valve types are better--mechanical or biological prostheses? Heart Lung Circ. 23: 1175-8.

Vahanian A, Alfieri O, Andreotti F, et al. 2012. Guidelines on the management of valvular heart disease (version 2012). eur heart J. 33:2451-96.

Van Nooten GJ, Caes FL, Franc sois KJ, et al. 1995. The valve choice in tricuspid valve replacement: 25 Years of experience. Eur J Cardiothorac Surg. 9:441-6. Discussion 446-7.

Wiedemann D, Rupprechter V, Mascherbauer J, et al. 2018. Tricuspid valve replacement: results of an orphan procedure - which is the best prosthesis? J Cardiovasc Surg (Torino). 59(4):626-32. 